\title{
Jets or vortices - what flows are generated by an inverse turbulent cascade?
}

\author{
Anna Frishman ${ }^{1,2}$, Jason Laurie ${ }^{1,3}$ and Gregory Falkovich ${ }^{1,4}$ \\ ${ }^{1}$ Department of Physics of Complex Systems, Weizmann Institute of Science, Rehovot 76100 Israel \\ ${ }^{2}$ Princeton Center for Theoretical Science, Princeton University, Princeton, New Jersey 08544, USA \\ ${ }^{3}$ Mathematics Group, School of Engineering and Applied Science, Aston University, Birmingham, B4 7ET, UK \\ ${ }^{4}$ Institute for Information Transmission Problems, Moscow, 127994 Russia
}

(Dated: March 3, 2017)

\begin{abstract}
An inverse cascade-energy transfer to progressively larger scales-is a salient feature of twodimensional turbulence. If the cascade reaches the system scale, it creates a coherent flow expected to have the largest available scale and conform with the symmetries of the domain. In a doubly periodic rectangle, the mean flow with zero total momentum was therefore believed to be unidirectional, with two jets along the short side; while for an aspect ratio close to unity, a vortex dipole was expected. Using direct numerical simulations, we show that in fact neither the box symmetry is respected nor the largest scale is realized: the flow is never purely unidirectional since the inverse cascade produces coherent vortices, whose number and relative motion are determined by the aspect ratio. This spontaneous symmetry breaking is closely related to the hierarchy of averaging times. Long-time averaging restores translational invariance due to vortex wandering along one direction, and gives jets whose profile, however, can be deduced neither from the largest-available-scale argument, nor from the often employed maximum-entropy principle or quasi-linear approximation.
\end{abstract}

Introduction. An inverse cascade is a counter-intuitive process of self-organization of two-dimensional turbulence. In an infinite medium, the cascade creates vortices (vortex rings in curved space [? ]) of ever-increasing size, while in a finite domain it eventually forms a flow coherent in the entire system. That flow is expected to become universal, i.e. independent of the forcing, when forcing scale goes to zero, keeping the energy input rate finite. Predicting the form of the flow in various settings is one of the central problems of turbulence theory. There are three known approaches to address this issue. The first way is qualitative: to look for the flow with the largest available scale (e.g. a flow dominated by the Fourier modes with the smallest wavenumber). A quantitative way, a statistical equilibrium theory, considers a flow profile that maximizes entropy for a given vorticity distribution and energy [? ? ]. It is strictly applicable only in the absence of forcing, although deviations from the predictions have been observed even then, see e.g. [? ? ? ? ? ]. The third approach is to assume that turbulence is weak relative to the mean flow and employ a quasi-linear approximation; writing equations for the two-point correlation functions (of velocity or vorticity) to form a closed system [? ? ? ? ], or using a single-point reduced description of spatial fluxes (of energy, momentum, enstrophy) [? ? ]. The limitations of the quasi-linear approximation for atmospheric flows has been pointed out in [?].

Perhaps the simplest setting is a rectangle with periodic boundary conditions (a torus). The system is translation invariant along $x$ and $y$; any nonuniform mean flow breaks one of these symmetries or both. Flow on a torus may have either contractible streamlines corresponding to vortices or non-contractible streamlines corresponding to jets. Jets going around one side may be expected in a (non-square) rectangle where there is no symmetry be- tween directions. Indeed, a maximal-entropy analysis [? ], predicts two opposing jets (for zero total momentum) directed along the shorter side of the domain for large enough aspect ratios. The non-equilibrium steady state was analysed numerically in [? ] and compared to this prediction. It was asserted that the mean flow indeed transitions between two jets consisting solely of noncontractible loops for a rectangle, and a vortex dipole, containing contractible streamlines, for domains close to a square.

One may also try to explain the appearance of the two flow types via the largest scale argument: for an aspect ratio $l_{x}$ substantially different from one, the largest mode is two opposite jets along the short side. On the other hand, in a square box, the jets can be directed along either side and one may expect a superposition of two sets of jets, which would look like a vortex dipole [? ? ]. In this picture, jets are fundamental objects on a torus while the vortex dipole appears only near a degeneracy, when $l_{x} \approx 1$.

In fact, the vortex, created by an extended inverse cascade in a square box of size $L \times L$, cannot be represented as a superposition of jets [? ? ], since the analysis of spatial fluxes of energy and momentum gives the velocity profile $U(r) \propto$ Const. at the distance from the vortex center $r \ll L[$ ? ]; that corresponds to the streamfunction $\psi(r) \propto r$ which cannot be represented as a superposition of orthogonal jets, $\psi(x, y)=\phi(x)+\phi(y)$.

In the present work, we focus on the universal limit of small-scale forcing and the culmination of an extended inverse cascade in a rectangle. Our numerical modeling reveals how all the expectations are defied by Nature: there is no dichotomy between vortices and jets, which coexist for any aspect ratio. In fact, such vortices appeared also in [? ], but were interpreted as intermediate size fluctuations rather than as part of the mean flow [? 
]. For domains with a moderate $l_{x}$, we find two jets and a vortex dipole at zero velocity streamlines. When averaged over the shortest dynamical timescale, the mean flow seems to be close to a steady Euler solution (compare Fig. ?? and Fig. S3 in the supplementary). On longer timescales, the distance between the vortices in the dipole varies, due to the influence of both fluctuations and jets. The asymptotic state is sensitive to the value of very small uniform friction: as it decreases for a moderate $l_{x}$, an additional vortex, that can be of either sign, emerges. Then, the two same-sign vortices no longer lie directly between the two jets, but inside opposing jets, giving a non-zero mean velocity, that implies the flow cannot correspond to a steady Euler solution. When the dipole or the three vortex flow is averaged over long times, vortices are smeared into stripes, resulting in a two-jet mean flow. Decreasing the aspect ratio even further causes the appearance of additional vortices, and what is more interesting, additional jets, which persist under long-time averaging. We thus find that the first two approaches (largest-scale and maximal-entropy), to the extent they can be applied, give incorrect predictions. The third approach, the quasi-linear approximation, relies on the expectation that the mean flow will dominate over turbulent fluctuations. It requires a proper account of the flow geometry and averaging time, and describes correctly the interior of a circular vortex [?], but fails to describe the global mean flow in a rectangle: the zonal or long time average treats vortices as fluctuations making the latter strong, as our numerics show. Our work thus demonstrates that the principles of organization of an inverse cascade into a mean flow are currently lacking.

Jets and vortices. We consider an incompressible flow, $\nabla \cdot \mathbf{v}=0$, described by the two-dimensional NavierStokes equations for a fluid with unit density:

$$
\partial_{t} \mathbf{v}+(\mathbf{v} \cdot \nabla) \mathbf{v}=-\nabla p-\alpha \mathbf{v}+\nu \nabla^{2} \mathbf{v}+\mathbf{f},
$$

The force $\mathbf{f}$ acts in a narrow band of scales $l_{f} \ll L$. The energy injection rate is $\epsilon=\langle\mathbf{f} \cdot \mathbf{v}\rangle$. We assume that system-size eddies produced by an inverse cascade have turnover times much shorter than the time of frictional dissipation: $\delta \equiv \epsilon^{-1 / 3} L^{2 / 3} \alpha \ll 1$. Then the inverse cascade fed by our small-scale forcing reaches the system scale producing energy accumulation and mean flow generation. In the steady state at high Reynolds number, $R e=\epsilon^{1 / 3} l_{f}^{4 / 3} / \nu \gg 1$, most of the energy is dissipated by the friction of the mean flow, giving the mean velocity estimate $U \simeq \sqrt{\epsilon / \alpha}$, and the corresponding turnover time $\tau_{m}=\sqrt{\alpha L^{2} / \epsilon}$.

We numerically solve (??) in the vorticity formulation in a periodic rectangle of size $2 \pi l_{x} \times 2 \pi$ using the grid $512 l_{x} \times 512$ with uniform spacing in both directions. We implement a pseudo-spectral method using the $3 / 2$ dealiasing rule and time step using a fourth-order exponential time-difference algorithm. Our stochastic forcing implements the Euler-Maruyama method, where each
Fourier mode of the forcing is a complex Gaussian random variable, correlated up to the timestep $d t$ of our simulations (see the supplementary), with fixed amplitude equal to 0.1 in an annulus of width $99 \leq k<101$ (acting as an approximation to delta-correlated white noise). The forcing scale is defined as $l_{f}=2 \pi / k_{f}$ with $k_{f} \approx 100$. In order to provide as large as possible inertial range for the inverse cascade, we replace regular viscosity with hyper-viscosity $-\nu\left(-\nabla^{2}\right)^{p} \mathbf{v}$ with $p=8$ and $\nu=1 \times 10^{-36}$. Each simulation is run so that a statistical steady state is reached, verified by monitoring the total energy. We compute the energy dissipation rate via friction using it as a measure of the inverse energy flux and an estimate for $\epsilon$. All of our data analysis is performed in the statistical steady state.

We begin from $l_{x}<1$ to see if the emergent mean flow has two opposite jets parallel to $\hat{x}$, with all averaged quantities independent of $x$. We perform three simulations, denoted by A-C, with $\alpha=1 \times 10^{-4}$ and with different aspect ratios: $l_{x}=1 / 2, \delta=5.58 \times 10^{-3}(\mathrm{~A})$, $l_{x}=3 / 4, \delta=4.86 \times 10^{-3}(\mathrm{~B})$ and $l_{x}=1, \delta=4.39 \times 10^{-3}$ (C). The typical vorticity snapshot in a steady state reveals a surprising feature: large-scale coherent vortices in addition to jets, see Fig. ??(a). Considering the dynamical generation of the mean flow, the presence of vortices is natural: locally the inverse cascade tends to create vortices, and the anisotropy of the box is felt only when their size is comparable to $l_{x} L$. Once established, the opposite-signed vortices feed on the constantly created smaller vortices, counteracting the effect of dissipation.

Any meaningful discussion of the emerging mean flow and symmetries must address the averaging times. For $t \lesssim \tau_{m}$ the centres of the vortices are effectively pinned. Averaging on such timescales, the mean flow can be characterized by streamlines as presented in Fig. ??. Topologically, the mean flow consists of two distinct regions of contractible streamlines surrounding the centres of the two vortices. In between the two regions, a separatrix should be present. The positive vortex is at $\left(-l_{x} L / 4,-L / 4\right)$ in Fig. ??. The symmetry of the streamline pattern around the vortex center dictates that the separatrix would pass through $\left(l_{x} L / 4,-L / 4\right)$, which is a stagnation point due to periodicity. For a square box, the vortices are arranged in a diagonal lattice, with the second vortex located at $\left(l_{x} L / 4, L / 4\right)$ and the separatrix passing through the stagnation point $\left(-l_{x} L / 4, L / 4\right)$. A separatrix composed of two straight streamlines connecting the two stagnation points preserves the $x-y$ symmetry, and is therefore expected for a square box where $l_{x}=1$. For $l_{x}<1$, this symmetry is absent and there is no reason for the two stagnation points to lie on the same streamline. We thus expect the separatrix to split into two, giving rise to two regions of non-contractible streamlines, i.e. jets. We indeed observe this splitting for aspect ratios $1 / 2$ and $3 / 4$, as seen in Fig. ??. In the square box, we also find the $x-y$ symmetry spontaneously broken 
by a tiny splitting of the separatrix, creating an opening for weak jets.

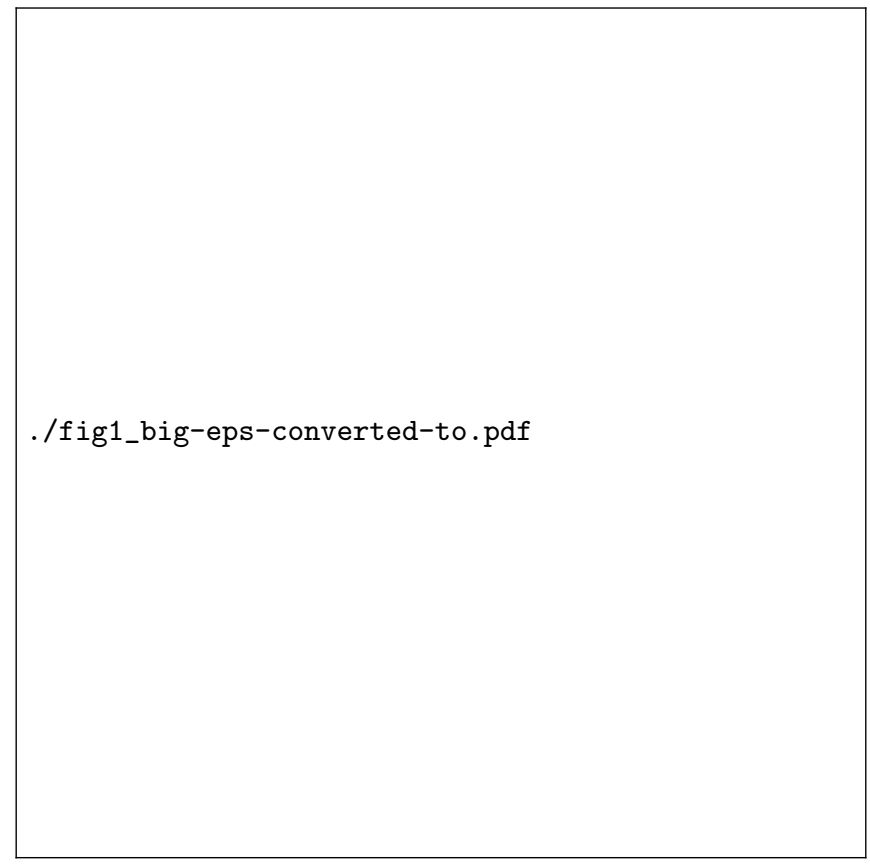

Figure 1. (color online) Scaled total vorticity $\sqrt{\alpha L^{2} / \epsilon}(\nabla \times \mathbf{v})$ for runs $\mathrm{A}$ and $\mathrm{F}$ respectively: (a) and (c) a snapshot, (b) and (d) a temporal average over time $1 / \alpha$ with frames shifted to align the velocity maximum with $y=0$.
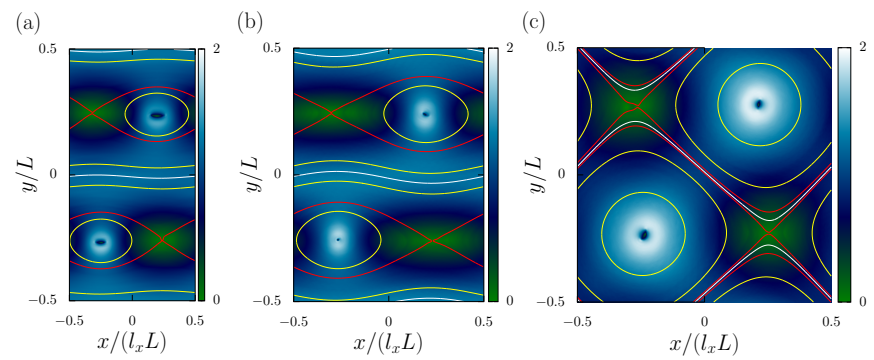

Figure 2. (color online) Heat maps of the scaled speed $(\sqrt{\alpha / \epsilon}|\mathbf{v}|)$ averaged over time $\tau_{m}$ : (a) run A, (b) run B, and (c) run C. Overlaid are streamlines, red lines are separatrices, white lines correspond to $\psi=0$.

On larger timescales, associated to the fluctuations $t \gtrsim \tau_{f} \equiv \epsilon^{-1 / 3} L^{2 / 3}$, a collective motion of the vortices, and a relative horizontal motion for $l_{x}<1$, becomes appreciable, see Fig. ?? and Fig. S4 in the supplementary. In particular, for $l_{x}=1 / 2$ and $l_{x}=3 / 4$, the diagonal lattice is only one among a continuum of possible configurations. In a square box, on the other hand, the vortices are almost completely restricted to the diagonal, separated by the maximal possible distance $L / \sqrt{2}$. This hints at the existence of a minimal distance between

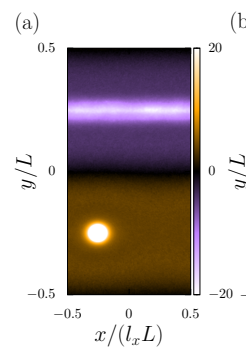

Figure 3 . (color online) Scaled total vorticity $\sqrt{\alpha L / \epsilon}(\nabla \times v)$ averaged over time $1 / \alpha$ in the reference frame of the positive vortex: (a) run $\mathrm{A}$, (b) run $\mathrm{B}$, and (c) run C.

./fig5-eps-converted-to.pdf
Figure 4. (color online) Mean profiles of vorticity and velocity (top), balances of energy (middle) and enstrophy (bottom) for run A (right) and run F (left). Horizontal dashed lines indicate the expected balance, solid lines indicate the numerical sum. Data were averaged over time exceeding $\tau_{d}$ with frames shifted to align the velocity maximum with $y=0$.

the vortex centers, which prevents the two vortices from approaching closer, dictating the extent of their relative motion. The fact that the vortices remain at the distance $L / \sqrt{2}$ for a square box suggests the guess that $l_{x} L / \sqrt{2}$ is the minimal vortex separation for an arbitrary aspect ratio $l_{x}$. Correspondingly, we find that the extent of the horizontal relative motion of the vortices for $l_{x}=3 / 4$ is close to $\left(l_{x}-\sqrt{2 l_{x}^{2}-1}\right) L$, see Fig. ??(b), see also Fig. S1 in the supplementary. This is in agreement with the absence of configurations with inter-vortex separations smaller than $l_{x} L / \sqrt{2}$, assuming that the vertical relative motion is negligible. Under the same assumption, the 
full extent of the relative horizontal motion should become possible for aspect ratios $l_{x}<1 / \sqrt{2}$. Indeed, as observed in Fig. ??(a) for $l_{x}=1 / 2<1 / \sqrt{2}$ the second vortex explores the line $y=L / 4$ with equal probability.

For $l_{x}=1 / 2$, we performed simulations for different $\delta: \delta=1.12 \times 10^{-2}(\mathrm{D}), \delta=2.78 \times 10^{-3}(\mathrm{E})$ and $\delta=1.67 \times 10^{-3}(\mathrm{~F})$. Astonishingly, the vortex dipole of simulations A and D is replaced by a three-vortex configuration for the low-friction simulations $\mathrm{E}$ and $\mathrm{F}$, see Fig. ??(a). In this configuration, the two same-sign vortices lie inside different jets, moving horizontally in opposite directions already on timescales of order $\tau_{m}$. The third vortex remains on the zero-velocity line between two jets. The distance between the opposite-sign vortices always exceeds the suggested minimal distance, $l_{x} L / \sqrt{2}$. In [?], it was argued that the size of the vortices should grow with decreasing $\delta$ for fixed $l_{f}$ and $\epsilon$, this contradicts the appearance of additional vortices found here.

How many vortices does the asymptotic $\delta \rightarrow 0$ state contain for a given $l_{x}$ ? If indeed there exists a minimal sustainable separation between opposite-sign vortices, then their number is limited by it. Assuming adjacent same signed vortices can appear only inside different jets, then at most four vortices, arranged in a diagonal lattice, can be present for $l_{x}=1 / 2$. The emergence of such a constricted arrangement out of the three-vortex configuration seems improbable. Thus, three vortices may be the asymptotic state for $l_{x}=1 / 2$.

Simulations $\mathrm{G}$ and $\mathrm{H}$ were done respectively for $l_{x}=$ $1 / 3, \delta=6.22 \times 10^{-3}$ and $l_{x}=1 / 4, \delta=6.83 \times 10^{-3}$. With decreasing aspect ratio, not only does the number of vortices increase but also the number of jets: four jets are present for $l_{x}=1 / 4$. This implies that the length of the short side of the box plays a crucial role in determining the jets, in contradiction to the largest-mode argument. Snapshots of the vorticity field are presented in Fig. S6 of the supplementary.

Long time average: mean flow and fluctuations. In the limit $\delta \rightarrow 0, \tau_{m}$ and $\tau_{d} \equiv 1 / \alpha$ become well separated and one can average over a time between them. For $l_{x}<1$, while there is random motion along $x$ on such timescales, almost no vertical (collective or relative) motion of the vortices is observable even for the longest times of order $\tau_{d}$, on which the square-box mean flow is close to zero, see Fig S5 in the supplementary. Thus, averaging over $t \gg \tau_{f} \gg \tau_{m}$ smears vortices into stripes resulting in an effective jet-like mean flow homogeneous in $x$, as is shown in Fig. ??(b) and Fig. ??(d) for $l_{x}=1 / 2$ (the relevance of such a situation for the ocean was suggested in [? ]). In Figs. ??(b) and ??(d) we align frames by the line of maximum (positive) $x$-averaged velocity. While the size of the vortices only slightly decreases with $\delta$, see Fig. ??, the averaged vorticity strip is narrower for smaller $\delta$ due to the suppression of the fluctuations which cause the vertical drift of the vortices.

The statistical homogeneity along $x$ at long times al- lows for an analysis of the energy and momentum balance similar to that in [? ]. Accordingly, we decompose the velocity into its mean $U(y)$ and fluctuating components: $\mathbf{v}=(u+U, v)$ where $u$ and $v$ are the corresponding fluctuating velocity fields in the $x$ and $y$ directions, and $\langle u\rangle=\langle v\rangle=0$, the average being over time. In the simulations a zonal average along $x$ is added. We write the steady state conservation of the $x$-momentum and energy neglecting the viscous terms, assuming $\nu / \alpha L^{2} \ll 1$ and $R e \rightarrow \infty$. Denoting $y$-derivatives by prime, one gets

$$
\begin{aligned}
\partial_{y}\langle u v\rangle+\alpha U & =0, \\
\partial_{y}\left\langle v\left(p+\frac{u^{2}+v^{2}}{2}\right)\right\rangle & =\epsilon-U^{\prime}\langle u v\rangle-\alpha\left\langle u^{2}+v^{2}\right\rangle .
\end{aligned}
$$

To make analytical progress, one usually assumes that fluctuations are suppressed by the mean flow and employs the quasi-linear approximation, neglecting the cubic terms in Eq. (??). The assumption seems to be supported both by the energy argument (most of the energy is transferred from fluctuations to the mean flow so one expects interactions of fluctuations to be unimportant) and by the momentum balance in Eq. (??) which gives $\langle u v\rangle \simeq \sqrt{\delta}(\epsilon L)^{2 / 3}$. For a circular vortex it was assumed additionally that the whole energy flux divergence is negligible [? ], including the pressure term. Using this assumption, Eq. (??) is reduced to $\epsilon=U^{\prime}\langle u v\rangle$, resulting in a closed system for $\langle u v\rangle$ and $U$ as $\delta \rightarrow 0$. Jets, however, have lines with $U^{\prime}=0$, where we expect the energy flux divergence to be comparable to $\epsilon$.

Figs. ??(a-b) show the mean flow profiles for simulations $\mathrm{A}$ and $\mathrm{F}$, and Figs. ??(c-d) present the terms of (??). To reduce noise, the data were low-pass filtered using a Gaussian kernel in Fourier space with an effective cutoff $L / 8$. Under long-time (or zonal) averaging, the vortices contribute both to the mean flow and the fluctuations, making them strong. The location of the vortices are thus characterized by peaks in the energy dissipation of the fluctuations.

For the dipole, the energy flux divergence is important everywhere, and in particular the cubic terms are not small. The flow thus cannot be described by a quasilinear approximation anywhere. In the three-vortex configuration, simulation F, the energy flux divergence is negligible in a small region between the same-sign vortices, the approximation of [? ] seems to be applicable there. The black curve in Figs. ??(c-d), representing the sum of terms in (??) without $\epsilon$, shows convergence of the statistics. Naturally, it is worse in the region of vortices where fluctuations are stronger.

It is also illuminating to consider the balance of enstrophy (squared vorticity) for the fluctuations:

$$
\frac{1}{2} \partial_{y}\left\langle v \omega^{2}\right\rangle=Q-\langle v \omega\rangle \Omega^{\prime},
$$

where $\omega$ and $\Omega=-U^{\prime}$ are the fluctuating and mean vorticity respectively, and $Q=\eta-\nu\left\langle\omega\left(-\nabla^{2}\right)^{p} \omega\right\rangle-\alpha\left\langle\omega^{2}\right\rangle$ 
with $\eta=\langle\omega(\nabla \times \mathbf{f}) \cdot \hat{z}\rangle$. Most of the injected enstrophy should be dissipated in the direct cascade by viscosity, so that when integrated over $y, Q$ is a small but finite rate of enstrophy absorption by the mean flow. The turbulenceflow enstrophy exchange term, $\langle v \omega\rangle \Omega^{\prime}=\alpha U \Omega^{\prime}$, turns into zero where $\Omega^{\prime}=0$ and $U=0$. That hints that the cubic term (turbulent enstrophy flux divergence) may be large there and the quasi-linear approximation invalid. Indeed, we find in Fig. ??(e-f) that the enstrophy balance is everywhere dominated by $Q$ and the turbulent flux, which goes from the jets to vortices, where viscous dissipation is larger. The quasi-linear approximation thus fails even in the regions where the velocity cubic terms are small in Fig. ??(c-d).

To conclude, let us reiterate the hierarchy of fluctuations and symmetries. The cascade-related weak fluctuations with velocities $v_{f} \simeq(\epsilon L)^{1 / 3}$ average to zero on a timescale exceeding their correlation time $\tau_{f}=L / v_{f}$. Vortices and jets have much larger velocities $U \simeq \sqrt{\epsilon / \alpha}$ and persist in the steady state. Fluctuations make the vortices wander along the jets, so averaging in a fixed reference frame over times exceeding $\tau_{f}$ (and hence exceeding their turnover time $L / U$ ) makes the flow unidirectional and restores the translation invariance along the short direction $x$. One may expect that averaging over even longer timescales gives zero mean flow and thus restores translational invariance along both $x$ and $y$. However, we found such a time only for a square box where the vortex dipole wanders around because the distance between vortices fluctuates. In the rectangle, the longest averages (over times exceeding $1 / \alpha$ ) give stable jets and thus do not restore the translation invariance along $y$. Apparently, it is much easier to move vortices or dipoles than it is to shift jets. Note also the remarkable breakdown of reflection symmetry by the appearance of a third vortex at lower friction.

This work provides only a first glimpse into the intricacies of flows created by an inverse cascade in a box with a globally broken $x-y$ symmetry. In geophysical systems one also has differential rotation ( $\beta$-effect), which breaks the symmetry locally and is expected to destroy the large-scale vortices. We leave the complete characterization of the parameter space $\delta, l_{x}, \beta$ for a future publication.

Acknowledgments. The authors are grateful to T. Grafke, V. Lebedev and P. Wiegmann for illuminating discussions. A.F. was supported by the Adams Fellowship Program of the Israel Academy of Sciences and $\mathrm{Hu}-$ manities. The work of G.F. was supported by the grant 882 of the Israel Science Foundation and the project 1422-00259 of the Russian Science Foundation.

A.F. and J.L. contributed equally to this work.
[] Gregory Falkovich and Krzysztof Gawedzki, "Turbulence on Hyperbolic Plane: The Fate of Inverse Cascade," J. Stat. Phys. 156, 10-54 (2014)

[] Jonathan Miller, "Statistical mechanics of Euler equations in two dimensions," Phys. Rev. Lett. 65, 2137-2140 (1990)

[] R. Robert and J. Sommeria, "Statistical equilibrium states for two-dimensional flows," J. Fluid Mech. 229, 291-310 (1991)

[] A. Venaille, T. Dauxois, and S. Ruffo, "Violent relaxation in two-dimensional flows with varying interaction range," Phys. Rev. E 92, 011001 (2015)

[] David G. Dritschel, Wanming Qi, and J. B. Marston, "On the late-time behaviour of a bounded, inviscid twodimensional flow," J. Fluid Mech. 783, 1-22 (2015)

[] Shun Ogawa, Julien Barré, Hidetoshi Morita, and Yoshiyuki Y. Yamaguchi, "Dynamical pattern formation in two-dimensional fluids and Landau pole bifurcation," Phys. Rev. E 89, 063007 (2014)

[] Hidetoshi Morita, "Collective oscillation in twodimensional fluid," arXiv:1103.1140 [cond-mat, physics:physics] (2011), arXiv: 1103.1140.

[] Patrick Tabeling, "Two-dimensional turbulence: a physicist approach," Phys. Rep. 362, 1-62 (2002).

[] Brian F. Farrell and Petros J. Ioannou, "Structure and Spacing of Jets in Barotropic Turbulence," J. Atmos. Sci. 64, 3652-3665 (2007)

[] Kaushik Srinivasan and W. R. Young, "Zonostrophic Instability," J. Atmos. Sci. 69, 1633-1656 (2011)

[] S. M. Tobias, K. Dagon, and J. B. Marston, "Astrophysical Fluid Dynamics via Direct Statistical Simulation," Astrophys. J. 727, 127 (2011).

[] F. Bouchet, C. Nardini, and T. Tangarife, "Stochastic averaging, large deviations and random transitions for the dynamics of $2 \mathrm{~d}$ and geostrophic turbulent vortices," Fluid Dyn. Res. 46, 061416 (2014).

[] Jason Laurie, Guido Boffetta, Gregory Falkovich, Igor Kolokolov, and Vladimir Lebedev, "Universal Profile of the Vortex Condensate in Two-Dimensional Turbulence," Phys. Rev. Lett. 113, 254503 (2014).

[] Gregory Falkovich, "Interaction between mean flow and turbulence in two dimensions," Proc. R. Soc. A 472, 20160287 (2016)

[] S. M. Tobias and J. B. Marston, "Direct Statistical Simulation of Out-of-Equilibrium Jets," Phys. Rev. Lett. 110, 104502 (2013)

[] Freddy Bouchet and Eric Simonnet, "Random Changes of Flow Topology in Two-Dimensional and Geophysical Turbulence," Phys. Rev. Lett. 102, 094504 (2009)

[] Freddy Bouchet and Antoine Venaille, "Statistical mechanics of two-dimensional and geophysical flows," Phys. Rep. 515, 227-295 (2012)

[] M. Chertkov, C. Connaughton, I. Kolokolov, and V. Lebedev, "Dynamics of Energy Condensation in TwoDimensional Turbulence," Phys. Rev. Lett. 99, 084501 (2007)

[] I. V. Kolokolov and V. V. Lebedev, "Structure of coherent vortices generated by the inverse cascade of twodimensional turbulence in a finite box," Phys. Rev. E 93, 033104 (2016)

[] Michael G. Schlax and Dudley B. Chelton, "The in- 
fluence of mesoscale eddies on the detection of quasi- zonal jets in the ocean," Geophys. Res. Lett. 35, L24602 (2008) 